\title{
The differences in short- and long-term varicella-zoster virus (VZV) immunoglobulin G levels following varicella vaccination of healthcare workers measured by VZV fluorescent-antibody- to-membrane-antigen assay (FAMA), VZV time-resolved fluorescence immunoassay and a VZV purified glycoprotein enzyme immunoassay
}

P. A. C. MAPLE ${ }^{1,2 *}$, J. HAEDICKE ${ }^{3}$, M. QUINLIVAN ${ }^{3}$, S. P. STEINBERG ${ }^{4}$, A. A. GERSHON ${ }^{4}$, K. E. BROWN ${ }^{1}$ AND J. BREUER ${ }^{3}$

${ }^{1}$ Virus Reference Department, Public Health England, Reference Microbiology Services, Colindale, London, UK

${ }^{2}$ East Yorkshire Microbiology, York Science Park, Heslington, York, UK

${ }^{3}$ Department of Infection, The Cruciform Building, University College London, London, UK

${ }^{4}$ Department of Pediatrics, Columbia University College of Physicians and Surgeons, New York, USA

Received 4 November 2015; Final revision 4 February 2016; Accepted 2 March 2016;

first published online 28 March 2016

\section{SUMMARY}

Healthcare workers (HCWs) reporting no history of varicella frequently receive varicella vaccination (vOka) if they test varicella-zoster virus (VZV) immunoglobulin $\mathrm{G}(\mathrm{IgG})$ negative. In this study, the utilities of VZV-IgG time-resolved fluorescence immunoassay (VZV-TRFIA) and a commercial VZVIgG purified glycoprotein enzyme immunoassay (gpEIA) currently used in England for confirming VZV immunity have been compared to the fluorescent-antibody-to-membrane-antigen assay (FAMA). A total of $110 \mathrm{HCWs}$ received two doses of vOka vaccine spaced 6 weeks apart and sera collected prevaccination $(n=100)$, at 6 weeks post-completion of vaccination $(n=86)$ and at $12-18$ months follow-up $(n=73)$ were analysed. Pre-vaccination, by FAMA, $61 \cdot 0 \%$ sera were VZV IgG negative, and compared to FAMA the sensitivities of VZV-TRFIA and gpEIA were $74 \cdot 4 \%$ [95\% confidence interval (CI) $57 \cdot 9$ 87.0] and $46 \cdot 2 \%(95 \%$ CI 30.1-62.8), respectively. Post-completion of vaccination the seroconversion rate by FAMA was $93.7 \%$ compared to rates of $95.8 \%$ and $70.8 \%$ determined by VZV-TRFIA and gpEIA, respectively. At 12-18 months follow-up seropositivity rates by FAMA, VZV-TRFIA and gpEIA were $78 \cdot 1 \%, 74 \cdot 0 \%$ and $47 \cdot 9 \%$, respectively. Compared to FAMA the sensitivities of VZV-TRFIA and gpEIA for measuring VZV IgG following vaccination were $96 \cdot 4 \%(95 \%$ CI $91 \cdot 7-98 \cdot 8)$ and $74 \cdot 6 \%(95 \%$ CI $66 \cdot 5-$ 81.6), respectively. Using both FAMA and VZV-TRFIA to identify healthy adult VZV susceptibles and measure seroconversion showed that vOka vaccination of HCWs is highly immunogenic.

Key words: Fluorescent-antibody-to-membrane-antigen-assay, healthcare workers, vOka vaccine, VZV glycoprotein EIA, VZV immunoglobulin G, VZV time-resolved fluorescence immunoassay.

\section{INTRODUCTION}

Primary infection with varicella zoster virus (VZV) manifests as varicella (chickenpox) and reactivation

\footnotetext{
* Author for correspondence: Dr P. A. C. Maple, East Yorkshire Microbiology, Innovation Centre, York Science Park, York YO10 5DG, UK.

(Email: eastyorksmicrobiol@gmail.com)
}

later in life produces herpes zoster (shingles). In the UK, historically, most cases of varicella occurred in the 5-14 years age group; however, in recent years there has been a sharp increase in the prevalence of varicella in the 1-4 years age group so that most cases are now reported in children aged $0-5$ years $[1,2]$. Primary varicella infection in healthy children is generally a mild, self-limiting disease which typically 
presents as a cropping vesicular rash; however, in immunocompromised individuals and susceptible adults, particularly pregnant women, the infection can be more serious and even life threatening [3-5].

An effective vaccine (vOka) against varicella has been available for a number of years and universal childhood vaccination $[6,7]$ is undertaken in a number of countries, e.g. Germany, Japan and the USA. The UK does not undertake universal varicella immunization and current UK policy [8] advocates vOka vaccination of significant contacts of immunocompromised individuals and healthcare workers (HCWs). Screening of HCWs through recall of history of chickenpox and laboratory testing to determine VZV IgG status in those with uncertain or negative histories followed by vOka vaccination of specific antibodynegative individuals is widely practised [9]. The use of highly sensitive assays to determine VZV IgG status is recommended [10].

The accepted gold standard assays for the determination of immunity/protection against VZV include the fluorescent-antibody-to-membrane-antigen assay (FAMA) [11, 12], and complement-enhanced VZV neutralization assay [13]. The FAMA and VZV neutralization assays are technically complex, subjective and unsuitable for testing large numbers of sera. Alternative, indirect immunofluorescence-based assays $[14,15]$ which are less technically demanding due to the use of glutaraldehyde or acetone-fixed infected cells are also available. Following the introduction of vOka vaccination [16] there has been a need for VZV IgG detection assays of high sensitivity because less VZV IgG is produced following vaccination compared to natural infection [17]. The Merck glycoprotein enzyme immunoassay (gpEIA; Merck EIA) was developed for this reason $[18,19]$ and was extensively evaluated, particularly in children [20]; however, the assay has extremely limited availability and is oversensitive in indicating immunity to varicella [21]. Commercial EIAs with purified VZV glycoproteins as antigen (gpEIAs) which differ methodologically from the Merck EIA are available; but, there are limited data comparing their performance in comparison with FAMA for measuring VZV IgG following vOka vaccination [22]. An 'in house' VZV IgG time-resolved fluorescence immunoassay (VZV-TRFIA) has also been developed using VZV-infected whole-cell lysate [23] and offers high analytical sensitivity and a large linear dynamic range due to the use of lanthanide chelates, which when coordinated with specific ligands and excited by ultraviolet absorption emit fluorescence for several hundreds of microseconds. Specific fluorescence is emitted over a much longer time period than background fluorescence and time-resolved measurement of fluorescence decay enables non-specific fluorescence which lasts a matter of nanoseconds to be excluded from the measured signal enabling highly sensitive and specific detection.

The immunization of adolescents or adults with vOka in a population where wild-type VZV freely circulates presents its own set of challenges; for instance, choosing appropriate assays to reliably identify VZV susceptibles in need of vaccination and then adequately measuring production of VZV IgG. In this study 110 HCWs with no reported history of chickenpox and testing VZV IgG negative, or equivocal, by a commercial non-glycoprotein-based EIA received two doses of vOka and their levels of VZV IgG at 6 weeks and 12-18 months post-second dose of vOka were measured. The utilities of VZV IgG TRFIA and a commercial gpEIA, which is recommended by UK Public Health England for testing VZV susceptibility, have been compared to FAMA.

\section{METHODS}

\section{Study population}

The serum samples $(n=259)$ used in this study were obtained from HCWs who were eligible for varicella vaccination as described previously [24]. The East London and the City Health Authority Local Research Ethics Committee (05/Q0605/1 granted ethical permission for the study. In brief, $110 \mathrm{HCW}$ s who tested VZV IgG negative, or equivocal, by a commercial assay (Diamedix ${ }^{\circledR}$, Diamedix Corporation, USA) received two doses of vOka vaccine (Sanofi Pasteur, Merck Sharp \& Dohme, France) 6 weeks apart. Serum samples available for this study were collected pre-vaccination $(n=100), 6$ weeks following the second vaccine dose $(n=86)$ and $12-18$ months following the second dose $(n=73)$. Sera were stored at $\leqslant-20^{\circ} \mathrm{C}$ prior to testing.

\section{FAMA}

VZV FAMA was performed using previously described methodology [11, 12]. MRC-5 cells were grown in Eagle's Minimum Essential Medium containing L-glutamine (Sigma, UK), supplemented with $10 \%$ fetal calf serum (FCS; Sigma, UK), $100 \times$ non-essential amino acids (Life Technologies, UK), $500 \times$ gentamicin and amphotericin B (Life 
Technologies, UK), and $1 \mathrm{ml}$ vancomycin at $5 \mathrm{mg} / \mathrm{ml}$ (Sigma, UK). Cells were infected at $90 \%$ confluence with an in-house wild-type VZV strain (THA). The multiplicity of infection was $0 \cdot 5$. Infected cells were harvested at $80-90 \%$ cytopathic effect by careful trypsinization, gently pelleted $(400 \mathrm{~g})$ and resuspended in an appropriate volume of phosphate buffered saline (PBS) for use fresh in setting up FAMA.

To set up the FAMA, $25 \mu$ l PBS was added to control and sample wells of a round-bottom 96well suspension culture plate (Grenier Bio-one, Germany). Control sera included VZV positive, intermediate positive, and negative sera diluted 1:2, 1:4 and 1:8 in consecutive wells. Serum negative, antibody negative and antibody/serum negative controls were employed in each run by replacing the respective components with $25 \mu \mathrm{l}$ PBS, except for the antibodynegative control to which a $1: 8$ dilution of the British standard VZV IgG antibody (90/690 NIBSC, UK) was added. A volume of $25 \mu \mathrm{l}$ of infected cell suspension was added to all wells and the plate incubated at room temperature for $30 \mathrm{~min}$. The cells were then washed three times with $100 \mu$ PBS with centrifugation at $400 \mathrm{~g}$ for $5 \mathrm{~min}$. A volume of $25 \mu \mathrm{l}$ of 1:50 dilution of polyclonal rabbit anti-human IgG FITC conjugate (Dako, UK) was added to all wells except the antibody-negative and antibody/serum-negative controls, and the plate was incubated at room temperature for 30 mins in the dark. Unbound antibody was removed by washing, as described previously and $10 \mu \mathrm{l}$ of $50 \%$ glycerol was added to each well. Volumes of $2 \mu \mathrm{l}$ from each well were transferred to a slide and visualized by confocal microscopy using a Zeiss AxioPlan 2 Microscope System (Campbell, USA). A bright fluorescent ring around the surface of infected cells indicated a sample positive for the presence of anti-VZV IgG. On average, between 10 and 20 infected cells were visualized per field and 10-20 fields examined depending upon the degree of fluorescence observed.

Results were reported as the lowest dilution at which distinct membrane fluorescence was visible. No membrane fluorescence at 1:2 dilution was graded as FAMA negative (susceptibility to $\mathrm{VZV}$ ), and fluorescence at 1:2, 1:4 or 1:8 was graded as FAMA positive (VZV protective). Typical fluorescence is shown in Figure 1.

\section{VZV-TRFIA}

DELFIA microtitre plates (PerkinElmer, UK) were coated with VZV ELISA grade antigen (Serion
Immundiagnostica $\mathrm{GmbH}$, Germany) at concentrations of $1 \cdot 0-2.0 \mu \mathrm{g} / \mathrm{ml}$ (depending on batch) prepared in $0.05 \mathrm{~m}$ carbonate/bicarbonate buffer ( $\mathrm{pH} \mathrm{9.6).} \mathrm{The}$ antigen was a sucrose-density gradient-purified extract of VZV strain Ellen grown in human embryo lung culture. The coating concentration of antigen selected was that which gave a Europium count of 400 000-600 000 with British Standard VZV antibody (NIBSC, UK) at a concentration of $50 \mathrm{mIU} / \mathrm{ml}$. The plates were stored overnight at $4{ }^{\circ} \mathrm{C}$ and washed four times with DELFIA wash buffer (PerkinElmer) using a DELFIA plate washer (PerkinElmer). Sera for testing were diluted 1:50 in DELFIA assay buffer (Perkin Elmer) and $100 \mu \mathrm{l}$ loaded into appropriate wells. A standard curve was run on each plate, prepared from British Standard VZV antibody diluted in DELFIA assay buffer at concentrations ranging from $50 \mathrm{mIU} /$ $\mathrm{ml}$ to $0.39 \mathrm{mIU} / \mathrm{ml}$. The plates were sealed and incubated in a humid chamber for $2 \mathrm{~h}$ at $37^{\circ} \mathrm{C}$ and then washed four times, as before. Europium-labelled antihuman IgG conjugate (PerkinElmer) diluted 1:500 in DELFIA assay buffer was added at $100 \mu \mathrm{l}$ per well using a multichannel pipette. The plates were then incubated for $1 \mathrm{~h}$ at $37^{\circ} \mathrm{C}$, washed four times, as before, and $150 \mu \mathrm{l}$ DELFIA enhancement solution (PerkinElmer) added to all wells. Following $10 \mathrm{~min}$ rotating incubation at room temperature, in the dark, the plates were read using a DELFIA 1234 reader (PerkinElmer) and data analysed using Multicalc software, v. 2000 (Wallac Oy, Finland). Interpolated antibody concentrations were expressed as $\mathrm{mIU} / \mathrm{ml}$.

In this study based on previous work [25] a cut-off VZV IgG level of $<100 \mathrm{mIU} / \mathrm{ml}$ has been used to indicate VZV susceptibility in non-vaccinated (prevaccination) adults and levels of VZV IgG $\geqslant 100$ $\mathrm{mIU} / \mathrm{ml}$ were considered protective. In adults vaccinated with vOka previous work [24] has shown that VZV IgG levels $>130 \mathrm{mIU} / \mathrm{ml}$ can be considered protective although relevant clinical studies are needed to confirm this assertion. Therefore the VZV-TRFIA cut-offs used in this study were $100 \mathrm{mIU} / \mathrm{ml}$ for HCWs pre-vaccination and $130 \mathrm{mIU} / \mathrm{ml}$ for HCWs post-vaccination.

\section{Commercial VZV gpEIA}

The commercial gpEIA used in this study was the VaccZyme $^{\mathrm{TM}}$ VZV glycoprotein IgG Low Level EIA kit supplied by The Binding Site (Birmingham, UK). The methodology for this assay has been described elsewhere [26] and the cut-off criteria used for the 

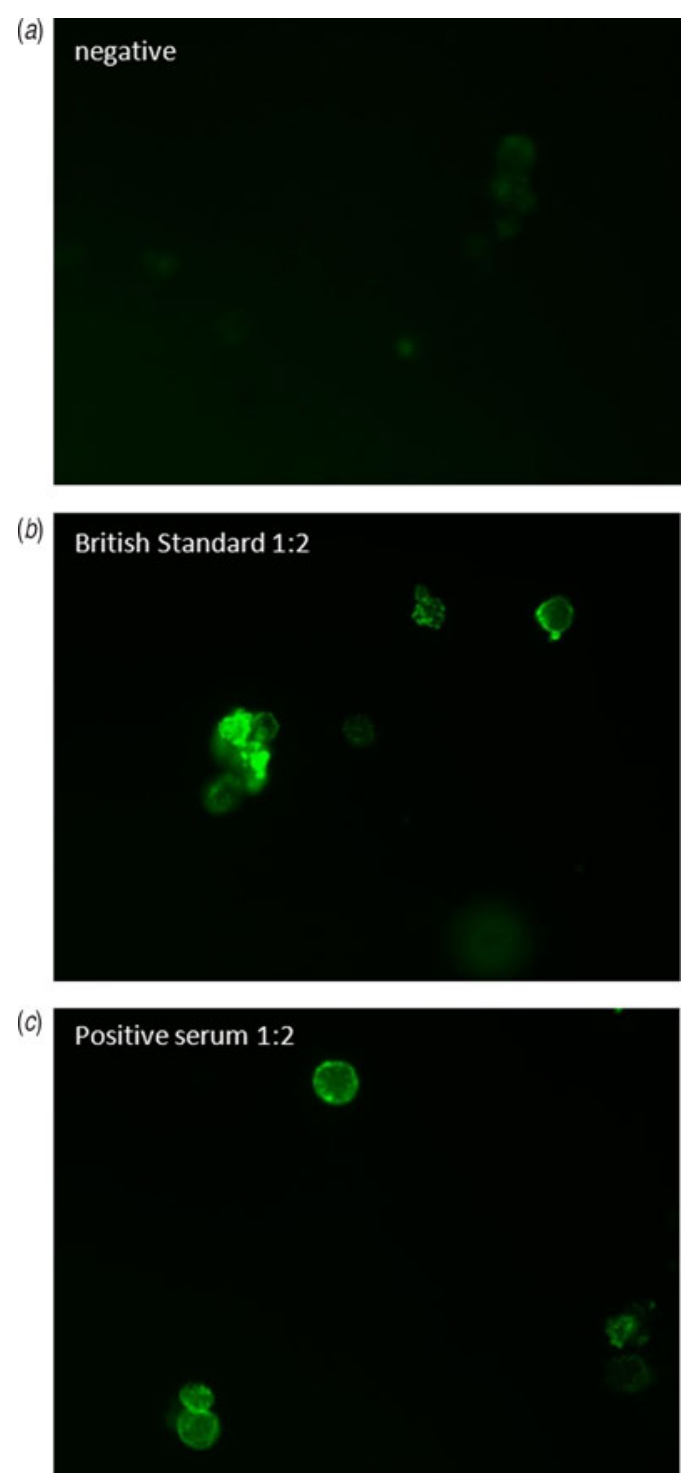

Fig. 1. Confocal microscopy $(20 \times$ objective, green fluorescent filter) of VZV-infected MRC-5 cells. (a) Exposed to VZV negative serum, (b) exposed to British Standard 90/690 VZV IgG and (c) exposed to serum from vOka-vaccinated individual. Note typical ring fluorescence in VZV IgG-positive sera.

purposes of this study were the same as those described above for the VZV-TRFIA. This commercial gpEIA has been used for a number of years by the Virus Reference Department, Colindale for confirmatory testing of VZV immunity status, particularly in pregnant women but is not recommended for assessing vaccine response [26].

\section{Assay variability}

It is widely recognized that FAMA is a subjective and technically demanding assay and to accommodate for this all FAMA work for this study was undertaken by a single author (J.H.). The competency of this worker and the reliability of the FAMA method set up at the Great Ormond Street Hospital, London, UK was checked by testing a proficiency panel of 64 serum samples (comprising pre-vaccination, post-vaccination and 12-18 months follow-up samples) which were then tested also by another author (S.P.S.) at the Columbia University College of Physicians and Surgeons, New York, USA, from where the reference FAMA originates. The agreement (kappa statistic $=$ $0 \cdot 4$ ) of the classification of the samples as either VZV IgG positive or negative using a cut-off titre of 2 was high with 94\% (44/47) of the samples tested giving concordant results. The sensitivity and specificity of the FAMA method used for this study was $93.9 \%$ and $92 \cdot 8 \%$ compared to the reference FAMA. Repeat testing of selected samples in separate FAMA runs also assessed the reproducibility of the FAMA methodology used for this study. Using FAMA, the frequency of samples tested with a $\geqslant$ fourfold difference in FAMA titres was 1:10-1:11.

The inter-assay variability (percentage coefficient of variation) of VZV-TRFIA in this study for a negative and low-positive sample was $12.5 \%$ and $10.9 \%$, respectively. Using the commercial gpEIA the interassay variability for a negative and low-positive sample was $4 \cdot 2 \%$ and $6 \cdot 2 \%$, respectively.

\section{Statistical analysis}

Sensitivity, specificity, positive predictive value (PPV) and negative predictive value (NPV) for VZV-TRFIA and the commercial gpEIA together with $95 \%$ confidence intervals (CIs) were calculated compared to FAMA overall and by visit. Results for the three assays were plotted against one another on log-scale axes to assess agreement and to calculate Pearson's correlation $(r)$. Results for the three assays were also plotted at each visit with geometric means and proportions $\geqslant 2$ by FAMA, $\geqslant 0 \cdot 1$ by TRFIA and $\geqslant 0 \cdot 1$ by gpEIA compared using a McNemar's test for paired data.

\section{RESULTS}

\section{Pre-vaccination VZV serological status of HCWs}

Results for FAMA, VZV-TRFIA and gpEIA were available for $100 / 110 \mathrm{HCWs}$, pre-vaccination. By FAMA (Table 1), 61.0\% sera had no VZV IgG 
detected, $16 \cdot 0 \%$ sera had FAMA titre $=2,13 \cdot 0 \%$ sera had FAMA titre $=4,7 \cdot 0 \%$ sera had FAMA titre $=8$ and $3.0 \%$ sera had FAMA titre $>8$.

A total of 57/61 (93.5\%) FAMA VZV IgG-negative sera tested VZV IgG negative by VZV-TRFIA and four $(6 \cdot 5 \%)$ sera tested VZV IgG positive. The mean VZV IgG level by VZV-TRFIA was $46 \cdot 2$ (range 15183) $\mathrm{mIU} / \mathrm{ml}$. Similarly, for the FAMA VZV IgGnegative sera there were 60/61 (98.4\%) sera which tested VZV IgG negative by commercial gpEIA and one $(1 \cdot 6 \%)$ serum which tested VZV IgG positive. The mean VZV IgG level by commercial gpEIA was 28 (range $<10-124$ ) $\mathrm{mIU} / \mathrm{ml}$.

For the FAMA VZV IgG-positive sera there were $29 / 39(74 \cdot 4 \%)$ sera that tested VZV IgG positive by VZV-TRFIA and $10(25 \cdot 6 \%)$ that were VZV IgG negative. The mean VZV IgG level by VZV-TRFIA was 356 (range 22-2507) $\mathrm{mIU} / \mathrm{ml}$. Similarly, for the FAMA VZV IgG-positive sera there were 18/39 (46.1\%) sera which tested VZV IgG positive by commercial gpEIA and $21(53.8 \%)$ sera which were VZV IgG negative. VZV IgG levels for the commercial gpEIA ranged between $<10$ and $>810 \mathrm{mIU} / \mathrm{ml}$.

Compared to FAMA the sensitivity and specificity of VZV-TRFIA at baseline was $74 \cdot 4 \%$ and $93 \cdot 4 \%$, respectively. Likewise, compared to FAMA the sensitivity and specificity of commercial gpEIA was $46 \cdot 2 \%$ and $100 \%$, respectively.

\section{Post-vaccination VZV serological status of HCWs 6 weeks following the second dose of vOka}

Results for FAMA, VZV-TRFIA and gpEIA were available for $86 / 110$ HCWs at 6 weeks postvaccination. By FAMA (Table 1), 5·8\% sera had no VZV IgG detected, 9.3 $\%$ sera had FAMA titre $=2$, $17 \cdot 4 \%$ sera had FAMA titre $=4,41.9 \%$ sera had FAMA titre $=8$ and $25.6 \%$ sera had FAMA titre $>8$. The mean VZV IgG level by VZV-TRFIA was $800 \cdot 9$ (range $78-3346$ ) $\mathrm{mIU} / \mathrm{ml}$. For the commercial gpEIA a mean VZV IgG value could not be computed as VZV IgG levels $>810 \mathrm{mIU} / \mathrm{ml}$ were not quantitated. Compared to FAMA the sensitivity of VZV-TRFIA for post-vaccine antibody was $100 \%$; however, specificity could not be reliably computed due to the low number $(n=5)$ of negative samples. Likewise, compared to FAMA the sensitivity of commercial gpEIA was $84 \cdot 0 \%$.

The rate of seroconversion in HCWs shown by FAMA to be VZV IgG negative at pre-vaccination was $93.7 \%$ as determined by FAMA compared to
$95 \cdot 8 \%$ as determined by VZV-TRFIA and $70 \cdot 8 \%$ as determined by commercial gpEIA.

\section{Post-vaccination VZV serological status of HCWs at 12-18 months follow-up}

Results for FAMA, VZV-TRFIA and gpEIA were available for $73 / 110 \mathrm{HCWs}$ at $12-18$ months followup. By FAMA (Table 1), 21.9\% sera had no VZV IgG detected, $13 \cdot 7 \%$ sera had FAMA titre $=2$, $26.0 \%$ sera had FAMA titre $=4,27 \cdot 4 \%$ sera had FAMA titre $=8$ and $11.0 \%$ sera had FAMA titre $>8$. By VZV-TRFIA and gpEIA there were 19 $(26.0 \%)$ and $38(52 \cdot 0 \%)$ sera, respectively, which tested VZV IgG negative. The mean VZV IgG level by VZV-TRFIA was 800.9 (range 78-3346) $\mathrm{mIU} / \mathrm{ml}$. For the commercial gpEIA a mean VZV IgG value could not be computed as VZV IgG levels $>810$ $\mathrm{mIU} / \mathrm{ml}$ were not quantitated.

There were $13 \mathrm{HCW}$ s who tested VZV IgG negative by FAMA at 12-18 months follow-up, despite being FAMA positive 6 weeks following the second dose of vOka. The serological profiles of these HCWs, who appeared to lose VZV IgG over the longer term according to FAMA, are shown in Table 2. Two HCWs appeared to lose VZV IgG which appeared to have been present pre-vaccination, one (study no. 1100) had a FAMA titre $=2$ and one (study no. 1046) had a FAMA titre $=4$. Compared to FAMA the sensitivity and specificity of VZV-TRFIA at 12 18 months post-immunization was $91 \cdot 2 \%$ and $87 \cdot 5 \%$, respectively and for commercial gpEIA the sensitivity and specificity was $61.4 \%$ and $100 \%$, respectively.

\section{Overall comparison of VZV-TRFIA and gpEIA with FAMA}

Both VZV-TRFIA and gpEIA correlated well with FAMA, with Pearson $r$ values of 0.84 and 0.83 , respectively. The VZV-TRFIA geometric mean VZV IgG level increased from $71 \mathrm{mIU} / \mathrm{ml}$ at prevaccination to $619 \mathrm{mIU} / \mathrm{ml}$ at 6 weeks postvaccination and then declined to $301 \mathrm{mIU} / \mathrm{ml}$ at 12-18 months follow-up (Table 1). Likewise, for FAMA the geometric mean VZV IgG titre increased from 1.7 to 6.6 and then decreased to 3.8 prevaccination, 6 weeks post-vaccination and 12-18 months follow-up, respectively. The commercial gpEIA geometric mean VZV IgG level increased from $23 \mathrm{mIU} / \mathrm{ml}$ at pre-vaccination to $315 \mathrm{mIU} / \mathrm{ml}$ at 6 weeks post-vaccination and then declined to 128 
Table 1. Results of FAMA, VZV-TRFIA and gpEIA at pre-vaccination, 6 weeks post-2 doses of vOka, and at 12-18 months follow-up

\begin{tabular}{|c|c|c|c|c|}
\hline Assay & Parameter & Pre-vaccination & $\begin{array}{l}6 \text { weeks post- } 2 \\
\text { doses vOka }\end{array}$ & $\begin{array}{l}\text { 12-18 months } \\
\text { follow-up }\end{array}$ \\
\hline \multirow{5}{*}{$\begin{array}{l}\text { FAMA numbers of sera } \\
\text { by titre per test group }\end{array}$} & Titre $<2$ & 61 & 5 & 16 \\
\hline & Titre 2 & 16 & 8 & 10 \\
\hline & Titre 4 & 13 & 15 & 19 \\
\hline & Titre 8 & 7 & 36 & 20 \\
\hline & Titre $>8$ & 3 & 22 & 8 \\
\hline FAMA titre/test group & Geometric mean titre & $1 \cdot 7$ & $6 \cdot 6$ & $3 \cdot 8$ \\
\hline VZV-TRFIA & Geometric mean VZV IgG (mIU/ml) & 71 & 619 & 301 \\
\hline Sensitivity & Value $\%(95 \% \mathrm{CI})$ & $74 \cdot 4(57 \cdot 9-87 \cdot 0)$ & $100(95 \cdot 5-100)$ & $91 \cdot 2(80 \cdot 7-97 \cdot 1)$ \\
\hline Specificity & Value $\%(95 \% \mathrm{CI})$ & $93 \cdot 4(84-98 \cdot 1)$ & $60 \cdot 0(14 \cdot 6-94 \cdot 7)$ & $87 \cdot 5(61 \cdot 6-98 \cdot 4)$ \\
\hline PPV & Value \% $(95 \% \mathrm{CI})$ & $87 \cdot 9(71 \cdot 8-96 \cdot 6)$ & $97 \cdot 6(91 \cdot 6-99 \cdot 7)$ & $96 \cdot 3(87 \cdot 2-99 \cdot 5)$ \\
\hline NPV & Value $\%(95 \% \mathrm{CI})$ & $85 \cdot 1(74 \cdot 3-92 \cdot 6)$ & $100(29 \cdot 2-100)$ & $73 \cdot 7(48 \cdot 8-90 \cdot 8)$ \\
\hline gpEIA & Geometric mean VZV IgG (mIU/ml) & 23 & 315 & 128 \\
\hline Sensitivity & Value \% $(95 \% \mathrm{CI})$ & $46 \cdot 2(30 \cdot 1-62 \cdot 8)$ & $84 \cdot 0(74 \cdot 1-91 \cdot 2)$ & $61 \cdot 4(47 \cdot 6-74)$ \\
\hline Specificity & Value $\%(95 \% \mathrm{CI})$ & $100(94 \cdot 1-100)$ & $80 \cdot 0(28 \cdot 4-99 \cdot 5)$ & $100(79 \cdot 4-100)$ \\
\hline PPV & Value $\%(95 \% \mathrm{CI})$ & $100(81 \cdot 5-100)$ & $98 \cdot 5(92 \cdot 2-100)$ & $100(90-100)$ \\
\hline NPV & Value $\%(95 \% \mathrm{CI})$ & $74 \cdot 4(63 \cdot 6-83 \cdot 4)$ & $23 \cdot 5(6 \cdot 8-49 \cdot 9)$ & $42 \cdot 1(26 \cdot 3-59 \cdot 2)$ \\
\hline
\end{tabular}

FAMA, Fluorescent-antibody-to-membrane-antigen; TRFIA, time-resolved fluorescence immunoassay; gpEIA, glycoprotein enzyme immunoassay; PPV, Positive predictive value; NPV, negative predictive value; CI, confidence interval.

$\mathrm{mIU} / \mathrm{ml}$ at $12-18$ months follow-up. The geometric mean VZV IgG levels detected by commercial gpEIA were significantly lower $(P<0 \cdot 01)$ compared to VZV-TRFIA. Based on comparison of the proportions of positive results, at pre-vaccination, postvaccination and $12-18$ months follow-up there was no significant difference $(P<0 \cdot 01)$ between VZVTRFIA and FAMA. The lower results by commercial gpEIA compared to FAMA and VZV-TRFIA were reflected in the quantitative and qualitative results (Table 1) with the difference in the proportion of positive results significantly lower at each visit $(P<0 \cdot 01)$.

Overall, compared to FAMA the combined sensitivity and specificity of VZV-TRFIA for detecting VZV IgG post-vaccination and at 12-18 months follow-up $(n=159)$ were $96 \cdot 4 \%(95 \%$ CI 91.7-98.8) with a PPV of $97 \cdot 0 \%(95 \%$ CI $92 \cdot 7-99 \cdot 2)$ and $80 \cdot 9 \%$ (95\% CI $58 \cdot 1-94 \cdot 5)$ with a NPV of $77 \cdot 3 \%(95 \%$ CI $54 \cdot 6-92 \cdot 2)$, respectively. Likewise, the sensitivity and specificity of commercial gpEIA for detecting VZV IgG postvaccination and at 12-18 months follow-up were $74 \cdot 6 \%(95 \%$ CI $66 \cdot 5-81 \cdot 6)$ with a PPV of $99 \cdot 0 \%$ (95\% CI 94.8-100) and $95 \cdot 2 \%(95 \%$ CI 76.2-99.9) with a NPV of $36 \cdot 4 \%(95 \%$ CI $23 \cdot 8-50 \cdot 4)$, respectively.

\section{DISCUSSION}

Few laboratories have been able to replicate the reference FAMA described by Gershon and colleagues; for example, Wutzler and colleagues [22, 27] when assessing the serological detection of VZV IgG by purified glycoprotein and whole cell lysate assays used an 'in house' modification of FAMA. Unfortunately, comparative data benchmarking their modified FAMA against the reference FAMA [11, 12] methodology does not appear to have been presented. A particular challenge with FAMA is the need to read slides while cells are still in suspension which is important to retain the presentation of functional epitopes. Technical expertise, subjectivity in reading, and interpretation of results are important issues with FAMA and to address these factors, in our study, a quality control test panel of 64 sera was jointly tested by author S.P.S. at New York and both sets of results were shown to be comparable. The FAMA described in our study showed $94 \%$ agreement with the reference FAMA performed at New York. FAMA, a technically demanding technique that is not easily automatable, is not suited for screening large numbers of samples although, recently, potential automation of the FAMA has been explored using a flow cytometry-adapted assay [28]. For small numbers of sera the high sensitivity and specificity of the visualization of membrane fluorescence confirms the utility of FAMA; however, according to our knowledge, there are no laboratories in the USA where FAMA is being performed.

This study highlights a number of potential pitfalls when estimating the response of adults to vOka 




vaccination. First, there is a need to reliably select adults who are truly non-immune to VZV. Selection of adult VZV susceptibles has been shown to lack specificity when using recall of a negative history of chickenpox although specificity can be improved by subsequent laboratory testing [29, 30]. The outcome of this approach is dependent on the sensitivity and specificity of laboratory test methodology used. In our study a commercial EIA using purified VZV whole cell extract was initially used to confirm VZV susceptibility in HCWs; however, 39\%, 29\% and $18 \%$ were VZV IgG positive by FAMA, VZV-TRFIA and commercial gpEIA, respectively. These findings underline the importance of using assays of similar sensitivity and specificity to FAMA if accurate determinations of those in need of vOka vaccination are to be made.

At 6 weeks following the second of two doses of vOka, $94 \cdot 2 \%$ of $\mathrm{HCW}$ tested positive for $\mathrm{VZV}$ IgG by FAMA. VZV-TRFIA correlated well with FAMA when applied to samples collected 6 weeks following the second dose of vOka with $100 \%$ of samples testing VZV IgG positive in both assays. The rate of seroconversion determined by FAMA was $93.7 \%$ and by VZV-TRFIA and commercial gpEIA was $95 \cdot 8 \%$ and $70 \cdot 8 \%$, respectively. The rates of seroconversion detected by FAMA (93.7\%) and VZVTRFIA $(95.8 \%)$ are consistent with that expected for immunocompetent adults receiving two doses of vOka 4-8 weeks apart [31].

Point cut-offs for VZV-TRFIA of VZV IgG of $93 \cdot 3$ $\mathrm{mIU} / \mathrm{ml}$ based on mixture modelling [32] and 100 $\mathrm{mIU} / \mathrm{ml}$ [25] based on protection following exposure to chickenpox have been proposed for VZV-TRFIA when applied to naturally infected populations. In this study a cut-off VZV IgG level of $100 \mathrm{mIU} / \mathrm{ml}$ has been used to grade HCWs pre-vaccination as either susceptible or immune and this approach is backed by recent guidance from Public Health England (www.phe.gov.uk, briefing note serial number 2015/067, 18 September, 2015). For VZVsusceptible individuals receiving vOka, a cut-off VZV IgG of $130 \mathrm{mIU} / \mathrm{ml}$ has been used in this study. The adoption of this cut-off is based on a previous study [24] in which avidity data was used to grade immune response to vOka as either primary or secondary and ROC analysis was used to generate a cut-off of VZV IgG of $130 \mathrm{mIU} / \mathrm{ml}$. The FAMA data generated in this study further justifies the use of a $130 \mathrm{mIU} / \mathrm{ml}$ cut-off for VZV immunity in adults following two doses of vOka. Two important caveats 
need to be applied as a consequence of the proposed adoption of the $130 \mathrm{mIU} / \mathrm{ml}$ cut-off. First, it should not be applied to children or immunocompromised adults as no data has been generated for these populations. Second, VZV-TRFIA should continue to be evaluated in vaccinees who develop breakthrough varicella after a known exposure. On the basis of the data gathered in this study we cannot recommend application for measuring response to vOka vaccination of the particular commercial gpEIA we have tested.

The immune response of adults compared to children is lower following vOka vaccination, which may be a consequence of a diminished capacity of circulating peripheral blood $\mathrm{T}$ lymphocytes to recognize VZV antigens after primary sensitization in vivo [33]. In our study, a significant reduction in VZV IgG antibody levels was seen at 12-18 months follow-up (Table 1). By FAMA, 78.1\% of HCWs had VZV IgG detected compared to $74.0 \%$ determined by VZV-TRFIA and $47.9 \%$ determined by gpEIA. The seropositivity rates determined by FAMA and VZVTRFIA are similar to those reported by Gershon and colleagues [34] who reported 94\% seropositivity 1-3 months after two doses of vaccine and $70 \%$ seropositivity by FAMA 2 years after the last dose of vaccine. Long-term serological data from immunized HCWs using methods other than FAMA or Merck gpEIA are limited. Saiman and colleagues have performed a similar study [35] to ours, in which sera from HCWs were tested by FAMA, commercially available latex agglutination assay and enzyme-linked immunosorbent immunoassay (ELISA). In comparison with FAMA, they reported the sensitivity and specificity of latex agglutination to be $82 \%$ and $94 \%$, respectively and ELISA demonstrated a sensitivity and specificity of $74 \%$ and $89 \%$, respectively.

In our study it has been shown in HCWs that for serum samples taken pre- and post-vOka vaccination there is good correlation between VZV IgG levels measured by VZV-TRFIA and FAMA. The lack of reliable commercially produced assays for measuring VZV IgG following vOka vaccination has resulted in a reliance on 'in house' assays which may be difficult to replicate outside their host laboratories. The disappointing results for the commercial gpEIA used in this study are specific to the assay used and modification [36] or application of other commercial gpEIAs may yield better outcomes. VZV-TRFIA is automatable and the reagents used are commercially available (www.perkinelmer.co.uk). VZV-TRFIA appears a suitable alternative to FAMA for measuring post- vaccination immunity and further studies are required to confirm a long-term correlate of protection.

\section{ACKNOWLEDGEMENTS}

This study was funded by the Wellcome Trust grant no. GR075427, J.B. receives additional funding from the NIHR UCL/UCLH Biomedical Research Centre. A.A.G. was in receipt of NIH grant R01DK093094. We thank Dr Nick Andrews, Statistics, Modelling and Economics Department, Health Protection Directorate, Public Health England, London, for the provision of statistical advice and analysis. We are also grateful to Julianne Brown, Virology Department, Great Ormond Street Hospital, London, for advice on FAMA methodology and the production of Figure 1.

\section{DECLARATION OF INTEREST}

A.A.G. has acted as a consultant for GlaxoSmith Kline and Merck for which she holds an additional service contract. The remaining authors declare no conflict of interest.

\section{REFERENCES}

1. Kudesia $\mathbf{G}$, et al. Changes in age related seroprevalence of antibody to varicella zoster virus: impact on vaccine strategy. Journal of Clinical Pathology 2002; 55: $154-155$.

2. Manikkavasagan G, et al. The epidemiology of chickenpox in UK 5-year olds: an analysis to inform vaccine policy. Vaccine 2010; 28: 7699-7705.

3. Harger $\mathbf{J H}$, et al. Risk factors and outcome of varicella-zoster virus pneumonia in pregnant women. Journal of Infectious Diseases 2002; 185: 422-427.

4. Tunbridge AJ, et al. Chickenpox in adults-clinical management. Journal of Infection 2008; 57: 95-102.

5. Daley AJ, Thorpe S, Garland SM. Varicella and the pregnant woman: prevention and management. Australian and New Zealand Journal of Obstetrics and Gynaecology 2008; 48: 26-33.

6. Breuer J. Vaccination to prevent varicella and shingles. Journal of Clinical Pathology 2001; 54: 743-747.

7. Bonanni $\mathbf{P}$, et al. Varicella vaccination in Europe - taking the practical approach. BMC Medicine 2009; 7: 26.

8. Breuer $\mathbf{J}$. Live attenuated vaccine for the prevention of varicella-zoster virus infection: does it work, is it safe and do we really need it in the UK? Journal of Medical Microbiology 2003; 52: 1-3.

9. Almuneef M, et al. Varicella zoster immunity in multinational health care workers of a Saudi Arabian hospital. American Journal of Infection Control 2003; 31: 375-381. 
10. Baracco GJ, et al. Clinical and economic impact of various strategies for varicella immunity screening and vaccination of health care personnel. American Journal of Infection Control 2015; 43: 1053-1060.

11. Williams V, Gershon A, Brunell PA. Serologic response to varicella-zoster membrane antigens measured by indirect fluorescence. Journal of Infectious Diseases 1974; 130: 669-672.

12. Gershon AA, Krugman S. Seroepidemiologic survey of varicella: value of specific fluorescent antibody test. Pediatrics 1975; 56: 1005-1008.

13. Grose C, Edmond BJ, Brunell PA. Complement enhanced neutralizing antibody response to varicella-zoster virus. Journal of Infectious Diseases 1979; 139: 432-437.

14. Zaia JA, Oxman MN. Antibody to varicella-zoster virus-induced membrane antigen: immunofluorescence assay using monodisperse glutaraldehyde fixed target cells. Journal of Infectious Diseases 1977; 136: 519-530.

15. Grandien M, et al. Determination of varicella immunity by the indirect immunofluorescence test in urgent clinical situations. Scandinavian Journal of Infectious Diseases 1976; 8: 65-69.

16. Arvin AM. Varicella vaccine - the first six years. New England Journal of Medicine 2001; 344: 1007-1009.

17. Ndumbe PM, Cradock-Watson J, Levinsky RJ. Natural and artificial immunity to Varicella Zoster virus. Journal of Medical Virology 1988; 25: 171-178.

18. Wasmuth EH, Miller WJ. Sensitive enzyme-linked immunosorbent assay for antibody to Varicella-Zoster virus using purified VZV glycoprotein antigen. Journal of Medical Virology 1990; 32: 189-193.

19. Hammond O, et al. The optimization and validation of the glycoprotein ELISA assay for quantitative varicellazoster virus (VZV) antibody detection. Journal of Medical Virology 2006; 78: 1679-1687.

20. Breuer J, Schmid DS, Gershon AA. Use and limitations of varicella-zoster virus-specific serological testing to evaluate breakthrough disease in vaccinees and to screen for susceptibility to varicella. Journal of Infectious Diseases 2008; 197 (Suppl. 2): S147-151.

21. Michalik DE, et al. Primary vaccine failure after 1 dose of varicella vaccine in healthy children. Journal of Infectious Diseases 2008; 197: 944-949.

22. Sauerbrei A, Wutzler P. Serological detection of varicella-zoster virus-specific immunoglobulin $G$ by an enzyme-linked immunosorbent assay using glycoprotein antigen. Journal of Clinical Microbiology 2006; 44: 3094-3097.

23. Chris Maple PA, et al. Performance characteristics of a quantitative, standardised varicella zoster IgG time resolved fluorescence immunoassay (VZV TRFIA) for measuring antibody following natural infection. Journal of Virological Methods 2009; 157: 90-92.

24. McDonald SLR, et al. Evaluation of the time resolved fluorescence immunoassay (TRFIA) for the detection of varicella zoster virus (VZV) antibodies following vaccination of healthcare workers. Journal of Virological Methods 2011; 172: 60-65.

25. Boxall EH, et al. Follow-up of pregnant women exposed to chicken-pox: an audit of relationship between level of antibody and development of chickenpox. European Journal of Clinical Microbiology and Infectious Diseases 2011; 30: 1193-1200

26. Maple PAC, et al. Comparison of a commercial varicella zoster glycoprotein $\mathrm{IgG}$ enzyme immunoassay with a reference time resolved fluorescence immunoassay (VZV TRFIA) for measuring VZV IgG in sera from pregnant women, sera sent for confirmatory testing and pre and post-vOka vaccination sera from healthcare workers. Journal of Clinical Virology 2012; 53: 201-207.

27. Sauerbrei A, et al. Evaluation of three commercial varicella-zoster virus IgG enzyme-linked immunosorbent assays in comparison to the fluorescent-antibodyto-membrane-antigen test. Clinical and Vaccine Immunology 2012; 19: 1261-1268.

28. Lafer MM, et al. Comparative study of the standard fluorescent antibody to membrane antigen (FAMA) assay and a flow cytometry-adapted FAMA assay to assess immunity to Varicella-Zoster virus. Clinical and Vaccine Immunology 2011; 18: 1194-1197.

29. Vandersmissen G, et al. Occupational risk of infection by varicella zoster virus in Belgian healthcare workers: a seroprevalence study. Occupational and Environmental Medicine 2000; 57: 621-626.

30. Holmes CN. Predictive value of a history of varicella infection. Canadian Family Physician 2005; 51: 60-65.

31. Hambleton S, Gershon AA. Preventing varicella-zoster disease. Clinical Microbiology Reviews 2005; 18: 70-80.

32. Maple PAC, et al. Performance of a time-resolved fluorescence immunoassay for measuring Varicella-Zoster virus immunoglobulin $\mathrm{G}$ levels in adults and comparison with commercial enzyme immunoassays and Merck glycoprotein enzyme immunoassay. Clinical and Vaccine Immunology 2006; 13: 214-218.

33. Nader S, et al. Age-related differences in cell-mediated immunity to varicella-zoster virus among children and adults immunized with live attenuated varicella vaccine. Journal of Infectious Diseases 1995; 171: 13-17.

34. Gershon AA, et al. Immunization of healthy adults with live attenuated varicella vaccine. Journal of Infectious Diseases 1988; 158: 132-137.

35. Saiman L, et al. Persistence of immunity to varicellazoster virus after vaccination of healthcare workers. Infection Control and Hospital Epidemiology 2001; 22: 279-283.

36. Kim YH, et al. Evaluation of a commercial glycoprotein enzyme-linked immunosorbent assay for measuring vaccine immunity to varicella. Yonsei Medical Journal 2014; 55: 459-466. 